\title{
Viscoelastic properties of maize starch/hydrocolloid pastes and gels
}

\author{
Martine Alloncle and Jean-Louis Doublier \\ INRA-LPCM, rue de la Géraudière, B.P. 527, 44026 Nantes Cedex 03, France
}

\begin{abstract}
Maize starch aqueous suspensions (concentration: 4\%) have been pasted in the presence of another polysaccharide (guar gum, locust bean gum or xanthan gum) at a concentration ranging from 0.1 to $0.5 \%$. The viscoelastic behaviour of these mixed systems has been investigated by performing harmonic measurements in three steps: (i) a mechanical spectrum at $60^{\circ} \mathrm{C}$, (ii) a gel-cure experiment at $25^{\circ} \mathrm{C}$ and (iii) a mechanical spectrum of the gel at $25^{\circ} \mathrm{C}$. The starch dispersion being described as a composite, its viscoelastic properties in the pasted and in the gelled state, as well, are primarily governed by the volume fraction occupied by the swollen particles which accounts for two-thirds of the total volume. The continuous phase makes an additional contribution due to its own viscoelastic properties. The added polysaccharide is concentrated within the continuous phase which makes it play an increasing role in the viscoelastic behaviour of the composite as the hydrocolloid content is increasing. More spectacular effects are experienced with xanthan gum than with the galactomannans in relation to the 'weak' gel properties of xanthan systems. Furthermore, the mechanical spectra of the mixed systems indicate that they are less elastic than with starch alone. Complex modifications in the starch dispersion are thus brought about by the hydrocolloid; these can be ascribed to phase separation processes in relation to incompatibility phenomena between unlike polysaccharides.
\end{abstract}

\section{Introduction}

Cereal starch/hydrocolloid mixtures are widely used because of their desirable effect on the texture and acceptability of foodstuffs. Starch in itself is employed as a thickening agent and it is well known that addition of an hydrocolloid (e.g. guar gum, locust bean gum, carrageenans, xanthan gum) strongly influences the viscosity of the hot starch paste (1-4). The origin of this synergistic effect has recently been explained on a rheological basis (4). It is generally accepted that cereal starch pastes can be described as suspensions of swollen particles dispersed in a macromolecular medium; the swollen particles consist mainly of amylopectin whereas the continuous phase contains predominantly amylose $(5,6)$. We recently demonstrated that adding the hydrocolloid results in a modification of the continuous phase where this polysaccharide remains located. As soon as gelatinization proceeds, starch granules swell, giving rise to a concentration of the hydrocolloid within the continuous phase. Due to the high viscosity and the dramatic concentration dependence of the hydrocolloid viscosity, this effect results in a spectacular rise in the viscosity of the continuous phase. The well-known synergistic effect in starch/hydrocolloid systems is thus explained on a simple rheological basis. Also, it is likely that starch gelation which takes place upon cooling and is known to be strongly influenced by the gelation of amylose is modified by the added polysaccharide. As the resulting continuous phase is an admixture of two polysaccharides, amylose and the added hydrocolloid, it is likely that immiscibility is exhibited, as has been shown for amylose/dextran and amylose/amylopectin mixtures $(7,8)$. In the present work we describe in more detail the rheological behaviour of corn starch/hydrocolloid 
systems. The added hydrocolloid is either a galactomannan (guar gum or locust bean gum) or a xanthan gum. Since dynamic rheological measurements allow the rheological parameters, $G^{\prime}$ and $G^{\prime \prime}$, to be instantaneously determined during gel-cure experiments, this technique is generally preferred to static (relaxation or creep) viscoelastic experiments which are time-dependent. The rheological properties were thus assessed using harmonic measurements in order to describe the properties of the hot paste, the kinetics of the gelation process which takes place upon cooling and the final gel properties.

\section{Materials and methods}

\section{Materials}

Commercial maize starch was obtained from Roquette Frères (Lestrem, France). The guar gum, locust bean gum and xanthan gum samples were supplied by Sanofi Bio-Industries (Carentan, France). Their physico-chemical characteristics are given in Table I.

\section{Procedures}

Galactomannan solutions were prepared by carefully sprinkling the gum onto the sides of a water vortex using a magnetic stirrer. Stirring was continued at low speed for $1 \mathrm{~h}$ at room temperature, then the solution was heated to $80^{\circ} \mathrm{C}$ for $0.5 \mathrm{~h}$ while stirring. Finally, the solution was filtered using sintered glass.

The xanthan solution was prepared in $\mathrm{KCl}(1 \%)$ using a comparable procedure unless the agitation was achieved by way of a motor-driven screwtype stirrer. Stirring was performed at room temperature for $2 \mathrm{~h}$ at 200 and then at 2500 r.p.m. for $5 \mathrm{~min}$.

The starch pastes were prepared using a 'high (heating) rate, low stirring rate' (HR-LS) procedure as described in (5). This consisted in heating the starch dispersion inside a double-walled, round-bottom vessel, stirring being achieved with an anchor-shaped blade rotating at 200 r.p.m. The maximum temperature $\left(96^{\circ} \mathrm{C}\right.$ ) was reached in $\sim 15 \mathrm{~min}$ and was held for $30 \mathrm{~min}$ before transferring to the rheological instrument. In all cases the starch powder was dispersed directly in the hydrocolloid solution which had been previously prepared. The pastes contained $4 \%$ starch and the hydrocolloid concentration varied from 0 (no hydrocolloid added) to $0.5 \%$. Exact concentrations of the blends were determined by drying aliquots overnight at $102^{\circ} \mathrm{C}$.

Table I. Physico-chemical characteristics of the hydrocolloid samples

\begin{tabular}{lllll}
\hline & $\begin{array}{l}{[\eta]} \\
(\mathrm{ml} / \mathrm{g})\end{array}$ & $\mathrm{M} / \mathrm{G}^{1}$ & $\begin{array}{l}\text { Acetyl content } \\
(\%)\end{array}$ & $\begin{array}{l}\text { Pyruvate content } \\
(\%)\end{array}$ \\
\hline $\begin{array}{l}\text { Guar gum } \\
\text { Locust bean gum }\end{array}$ & 1200 & 1.68 & - & - \\
Xanthan gum & 950 & 3.8 & - & - \\
\hline
\end{tabular}

${ }^{1}$ Mannose to galactose ratio.

${ }^{2} \mathrm{In} \mathrm{KCl}\left(0.13 \mathrm{~mol} / \mathrm{dm}^{3}\right)$. 


\section{Rheological measurements}

A controlled stress rheometer (CarriMed, CS 50) was used to perform oscillatory shear experiments using the cone and plate geometry $(5 \mathrm{~cm}$ diameter; $4^{\circ}$ cone angle). The strain amplitude was fixed at $4 \%$ after determination of the linearity limits which were found to be $\sim 6 \%$ for the paste at $60^{\circ} \mathrm{C}$ and the gel at $25^{\circ} \mathrm{C}$. Each mixed system was characterized in three steps. First, a mechanical spectrum $\left(G^{\prime}, G^{\prime \prime}\right.$ as a function of frequency from 0.01 to $\left.4 \mathrm{~Hz}\right)$ was plotted at $60^{\circ} \mathrm{C}$. This temperature allowed the hot paste to be characterized. The temperature was then rapidly decreased to $25^{\circ} \mathrm{C}$. Owing to the Peltier effect of the rheometer, this required only a few minutes. As soon as $25^{\circ} \mathrm{C}$ was reached, the $G^{\prime}$ and $G^{\prime \prime}$ evolution (at $1 \mathrm{~Hz}$ ) was recorded as a function of time for $15 \mathrm{~h}$ in order to describe the kinetics of the gelling process. Finally, a mechanical spectrum of the gelled system was established as in the first step.

\section{Results}

The mechanical spectra of the hot pastes at $60^{\circ} \mathrm{C}$ are given in Figures 1,2 and 3 for the blends with guar gum, locust bean gum and xanthan gum respectively. In each figure we show three spectra corresponding to starch alone (dashed lines), and two mixtures, 0.1 and $0.5 \%$ respectively. The other hydrocolloid concentrations we investigated, namely 0.2 and $0.3 \%$, lay in between. For starch alone, the storage modulus $\left(G^{\prime}\right)$ remained almost constant within the frequency range $0.1-4 \mathrm{~Hz}$, whereas the loss modulus $\left(G^{\prime \prime}\right)$ smoothly increased with frequency. Moreover, the $G^{\prime \prime} / G^{\prime}$ ratio was between $\sim 0.1$ at $0.1 \mathrm{~Hz}$ and $\sim 0.2$ at $5 \mathrm{~Hz}$. These characteristics respond to the definition of 'weak' gels as popularized by Clark and Ross-Murphy (9). As seen in Figure 1 for the mixtures with guar gum,

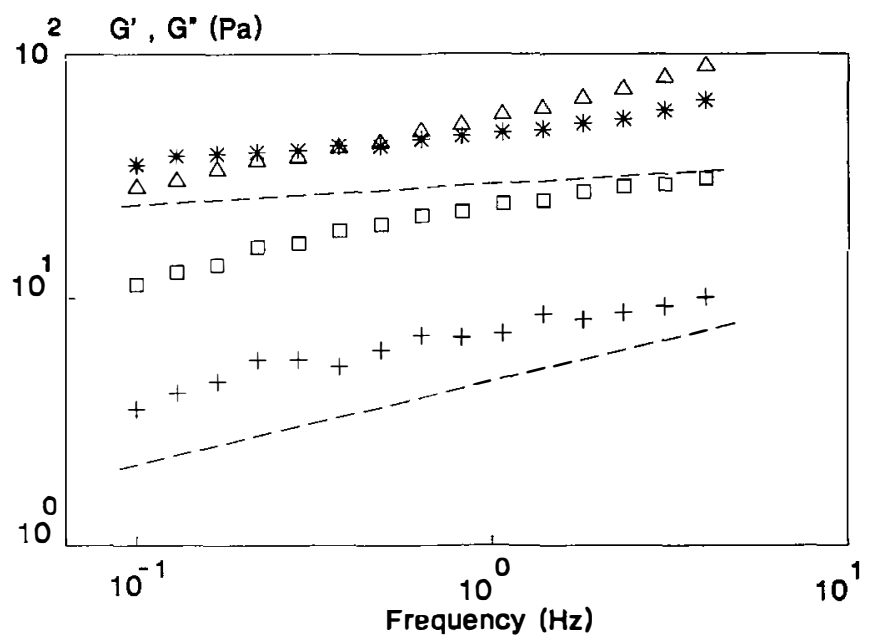

Fig. 1. Mechanical spectra of starch/guar gum mixtures. Dashed lines, starch (concentration $4 \%$ ). Starch $4 \%$ /guar gum $0.1 \%\left(*, G^{\prime} ;+, G^{\prime \prime}\right)$; starch $4 \%$ /guar gum $0.5 \%\left(\triangle, G^{\prime} ; \square, G^{\prime \prime}\right)$. Temperature, $60^{\circ} \mathrm{C}$. 
addition of the hydrocolloid had an effect on $G^{\prime}$ and $G^{\prime \prime}$. With the $0.1 \%$ mixture, $G^{\prime \prime}$ was slightly increased and paralleled quite closely the $G^{\prime \prime}$ trace of starch alone. On the other hand, $G^{\prime}$ was increased $(\times \sim 1.7)$ but, more spectacularly, the frequency dependence of this modulus was increased, the slope of the $G^{\prime}$ versus frequency curve being 0.31 compared with 0.12 for starch alone. These trends were accentuated as the guar content increased: $G^{\prime \prime}$ trended to $1 \mathrm{~Pa}$ at $0.1 \mathrm{~Hz}$ at a $0.5 \%$ guar content against $0.2 \mathrm{~Pa}$ for starch alone.

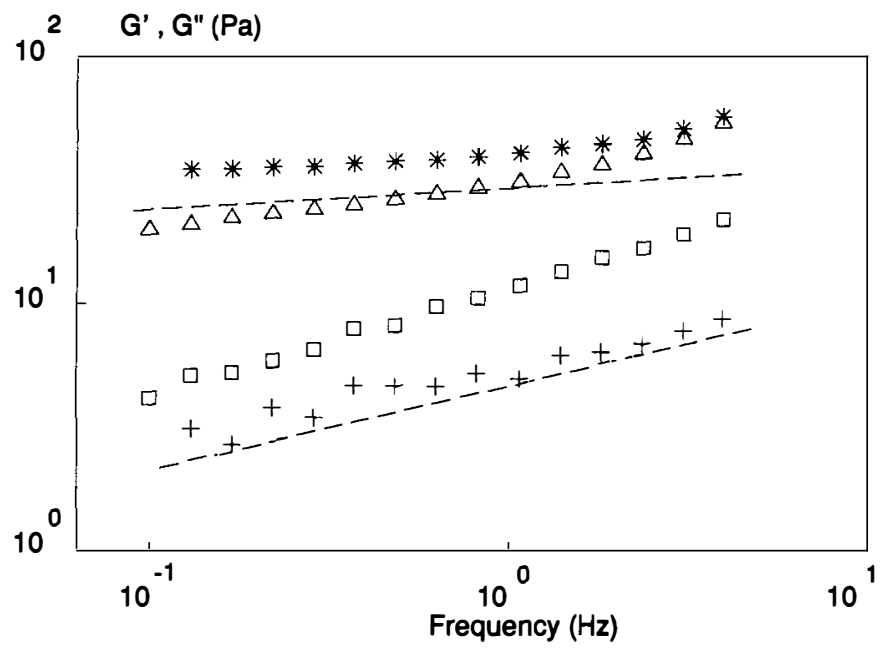

Fig. 2. Mechanical spectra of starch/locust bean gum mixtures. Dashed lines, starch (concentration, $4 \%)$. Starch $4 \% /$ locust bean gum $0.1 \%\left(*, G^{\prime} ;+, G^{\prime \prime}\right)$; starch $4 \% /$ locust bean gum $0.5 \%\left(\triangle, G^{\prime} ; \square\right.$, $\left.G^{\prime \prime}\right)$. Temperature, $60^{\circ} \mathrm{C}$.

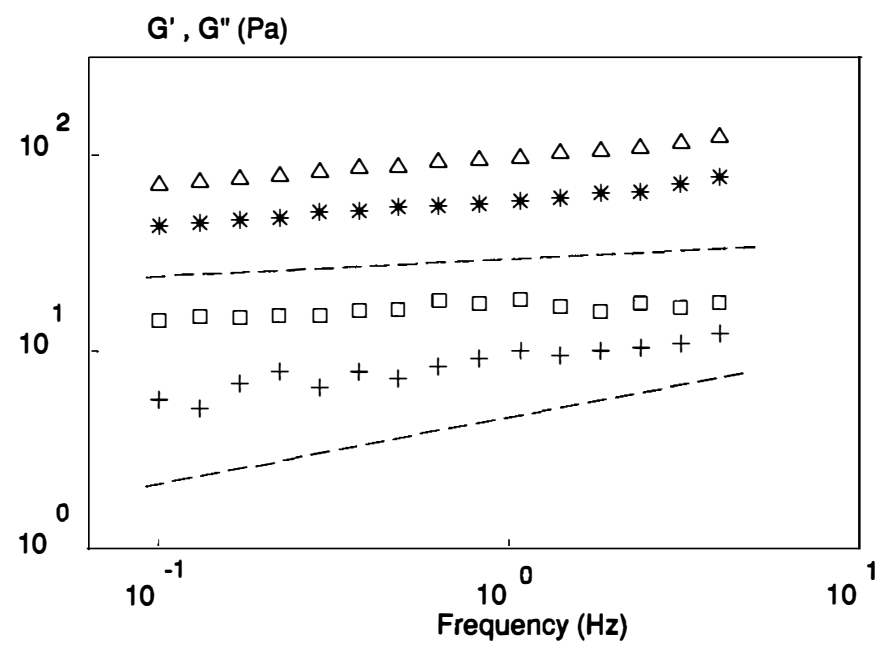

Fig. 3. Mechanical spectra of starch/xanthan gum mixtures. Dashed lines, starch (concentration, $4 \%)$. Starch $4 \% / x a n t h a n$ gum $0.1 \%\left(*, G^{\prime} ;+, G^{\prime \prime}\right)$; starch $4 \% / x a n t h a n$ gum $0.5 \%\left(\triangle, G^{\prime} ; \square, G^{\prime \prime}\right)$. Temperature, $60^{\circ} \mathrm{C}$. 
Moreover, $G^{\prime}$ became more frequency-dependent, the slope of the $G^{\prime}$ versus frequency curve increasing to 0.5 . In contrast, the $G^{\prime}$ value remained of the same order as for the $0.1 \%$ mixture. Quite similar patterns were seen with starch/locust bean gum blends. However, the effects appeared to be less pronounced. Incorporation of locust bean gum at $0.1 \%$ resulted in higher values of $G^{\prime}$ whereas $G^{\prime \prime}$ could not be differentiated from $G^{\prime \prime}$ of starch alone. On the other hand, $G^{\prime}$ was lower than at $0.1 \%$ for the $0.5 \%$ mixture and was of the same order as for starch alone. Meanwhile, $G^{\prime \prime}$ was slightly increased. Here also the main effect was related to the frequency-dependence of $G^{\prime}$ with a slope of the $G^{\prime}$ (frequency) curve, which was 0.4 against 0.16 at $0.1 \%$ of locust bean gum. As seen in Figure 3, the effects resulting from the incorporation of xanthan gum were spectacularly different. Higher values of $G^{\prime}$ and $G^{\prime \prime}$ were seen as the xanthan content increased. It can also be noticed that the slope of the $G^{\prime}$ (frequency) curves did not change while the $G^{\prime \prime}$ versus frequency tended to level off. Figure 4 shows the $G^{\prime}$ and $G^{\prime \prime}$ variations at $1 \mathrm{~Hz}$ as a function of concentration. This provides a clear illustration of the above-mentioned effects. A quasi-linear dependency of $G^{\prime}$ and $G^{\prime \prime}$ upon concentration is seen with xanthan gum. In contrast, more complex variations are observed for the galactomannans with a slight increase of $G^{\prime}$ for gụar gum and a downward curvature of the $G^{\prime}$ versus concentration curve with a maximum at $0.2 \%$ for locust bean gum. Meanwhile, the $G^{\prime \prime}$ versus concentration curves were linear but the concentration dependence remained slight.

The kinetics of gel formation of starch-guar blends are compared to starch alone in Figure 5. For the sake of clarity only two mixtures are presented (guar: 0.1 and $0.5 \%)$. Similar trends were observed for the other guar concentrations $(0.2$ and $0.3 \%)$. For starch alone, the storage modulus increased steadily for the

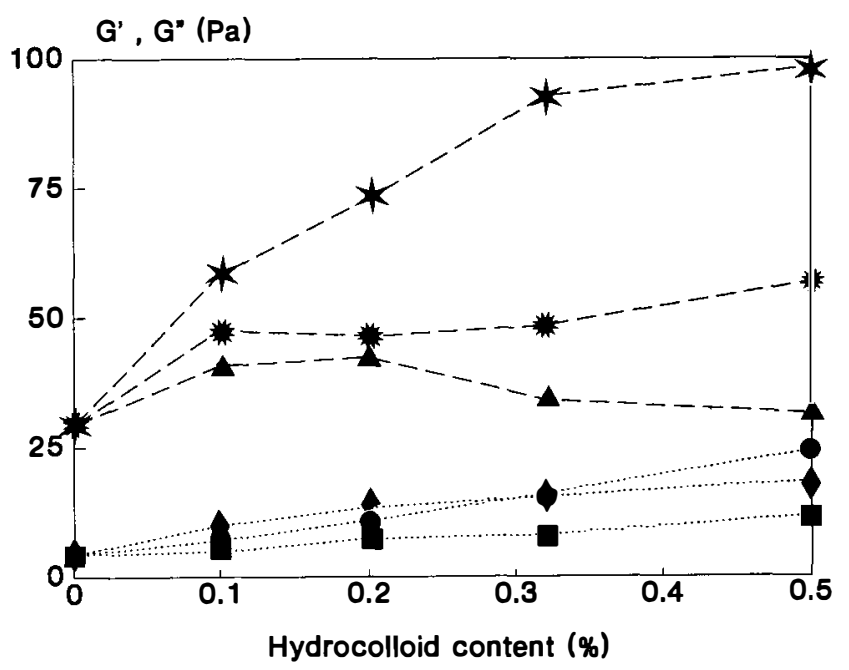

Fig. 4. $G^{\prime}$ and $G^{\prime \prime}$ variations as a function of concentration. Temperature, $60^{\circ} \mathrm{C}$. Frequency, $1 \mathrm{~Hz}$, guar gum $\left(*, G^{\prime} ; \boldsymbol{\bullet}, G^{\prime \prime}\right)$. Locust bean gum $\left(\Delta, G^{\prime} ; \boldsymbol{\square}, G^{\prime \prime}\right)$. Xanthan gum $\left(\star, G^{\prime} ; \bullet, G^{\prime \prime}\right)$. 


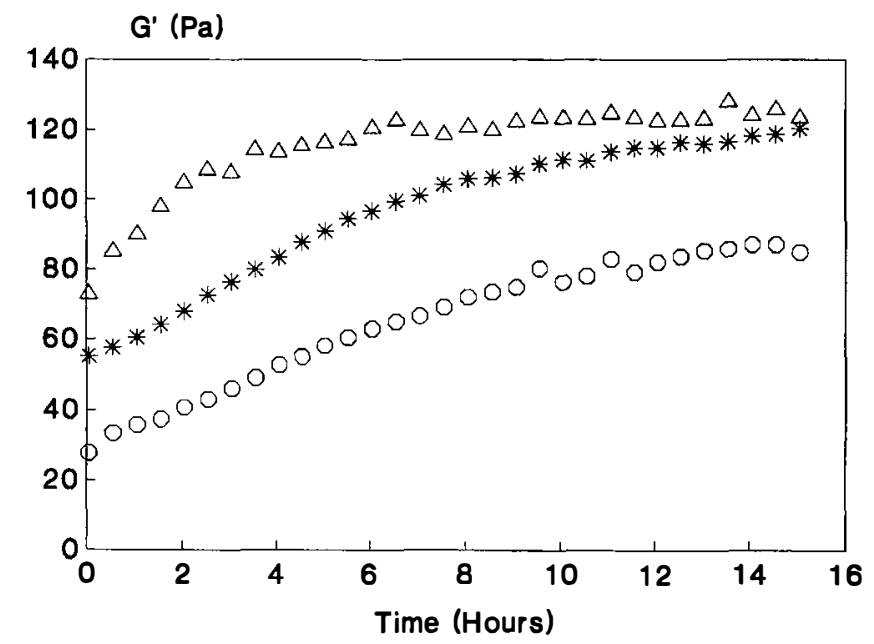

Fig. 5. $G^{\prime}$ variations as a function of time. Temperature, $25^{\circ} \mathrm{C}$. Starch concentration, $4 \%$. Guar gum concentration: $(\bigcirc) 0 \%,(*) 0.1 \% ;(\triangle) 0.5 \%$.

first $12 \mathrm{~h}$ from $\sim 28$ to $\sim 80 \mathrm{~Pa}$ and then remained constant. The patterns displayed by the starch-guar mixtures were quite different: the initial $G^{\prime}$ values were of $\sim 50$ and $\sim 70 \mathrm{~Pa}$ for the 0.1 and $0.5 \%$ guar contents respectively. In the two blends $G^{\prime}$ increased steadily as a function of time and then levelled off. The first period was shortened, lasting $\sim 6 \mathrm{~h}$ for the less concentrated system (guar: $0.1 \%$ ) and $\sim 3 \mathrm{~h}$ for the concentrated one (guar: $0.5 \%$ ). The final $G^{\prime}$ value also was higher than for starch alone, typically $\sim 110-120$ versus $80 \mathrm{~Pa}$.

Figure 6 shows the results obtained in similar conditions for the kinetics of gelation of starch-locust bean gum mixtures. Three concentrations are shown here, namely $0.1,0.2$ and $0.5 \%$. The initial value of $G^{\prime}$ was $\sim 40-50 \mathrm{~Pa}$, that is not so high as with guar gum. Furthermore, the initial $G^{\prime}$ values did not vary when the locust bean gum concentration increased. For the $G^{\prime}$ versus time variations marked differences were seen, depending upon the amount of locust bean gum added. At the lower locust bean gum content the kinetics appeared to be slightly slower than for starch alone and the final $G^{\prime}$ value $(\sim 85 \mathrm{~Pa})$ did not differ very much. For an intermediate concentration $(0.2 \%)$, a similar pattern as with starch-guar mixtures was observed with a first period (the first $8 \mathrm{~h}$ ) characterized by a rapid $G^{\prime}$ increase followed by a levelling at longer times. The final $G^{\prime}$ value was $\sim 100 \mathrm{~Pa}$.

For the more concentrated mixture $(0.5 \%)$, the first period was shorter $(\sim 3 \mathrm{~h})$, as with guar, and the final rigidity of the gel appeared slightly less than for starch alone ( $\sim 75$ versus $\sim 90 \mathrm{~Pa})$.

Results obtained with xanthan gum are quite different, as illustrated in Figure 7. Three concentrations are shown, together with starch alone for a comparison. The $G^{\prime}$ versus time traces of the mixtures roughly paralleled that of starch alone with higher moduli, the higher the xanthan content, the higher the $G^{\prime}$ value. 


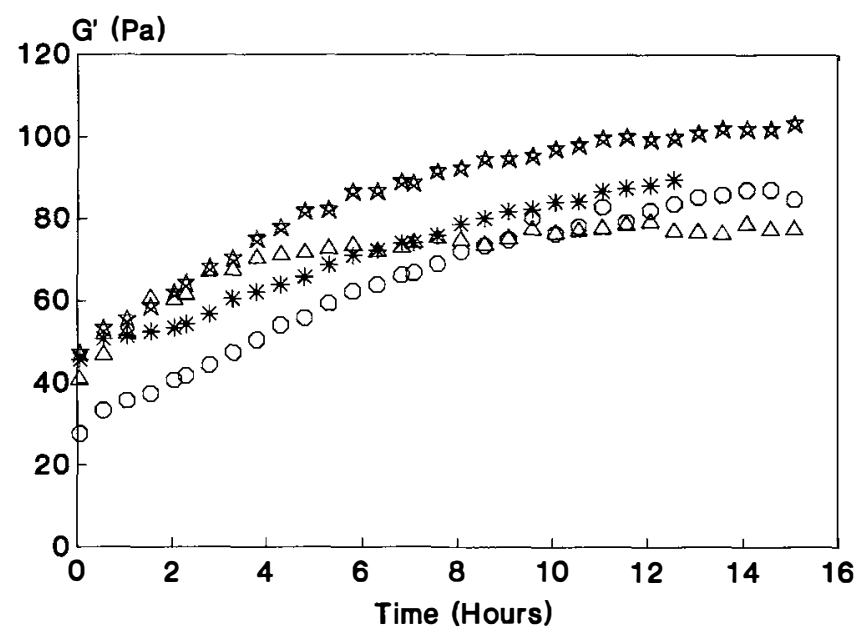

Fig. 6. $G^{\prime}$ variations as a function of time. Temperature, $25^{\circ} \mathrm{C}$. Starch concentration, $4 \%$. Locust bean gum concentration: $(\bigcirc) 0 \% ;(*) 0.1 \%$; $(\star) 0.2 \% ;(\triangle) 0.5 \%$.

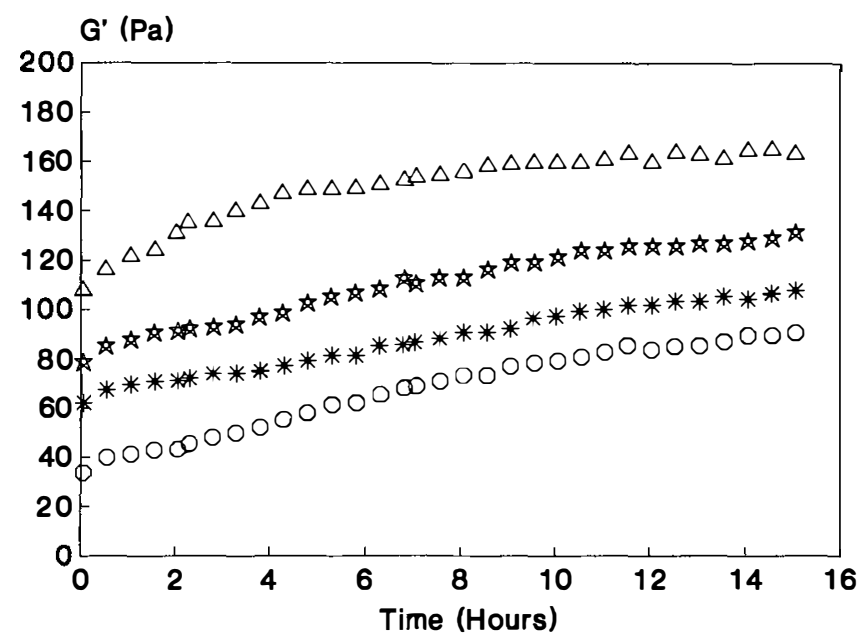

Fig. 7. $G^{\prime}$ variations as a function of time. Temperature, $25^{\circ} \mathrm{C}$. Starch concentration, $4 \%$. Xanthum gum concentration: $(\bigcirc) 0 \% ;(*) 0.1 \%$; $(\star) 0.2 \%$; $(\triangle) 0.5 \%$.

However, for the $0.5 \%$ concentration, the first period also was shortened to $\sim 3 \mathrm{~h}$ as with the galactomannans.

Figures 8,9 and 10 show the mechanical spectra of the final gels. Starch alone resulted in a classical pattern for a true gel according to the 'operational' definition, with the well-known flat frequency-dependence and $G^{\prime}>10 G^{\prime \prime}(9)$. The low guar concentrated blend exhibited a pattern quite similar to starch unless the two moduli were slightly higher. In contrast, for the $0.5 \%$ mixture, $G^{\prime}$ had become frequency-dependent with a slope, in logarithmic scales, of $\sim 0.16$, while $G^{\prime \prime}$ varied much more, the slope being $\sim 0.5$ within the low frequency 


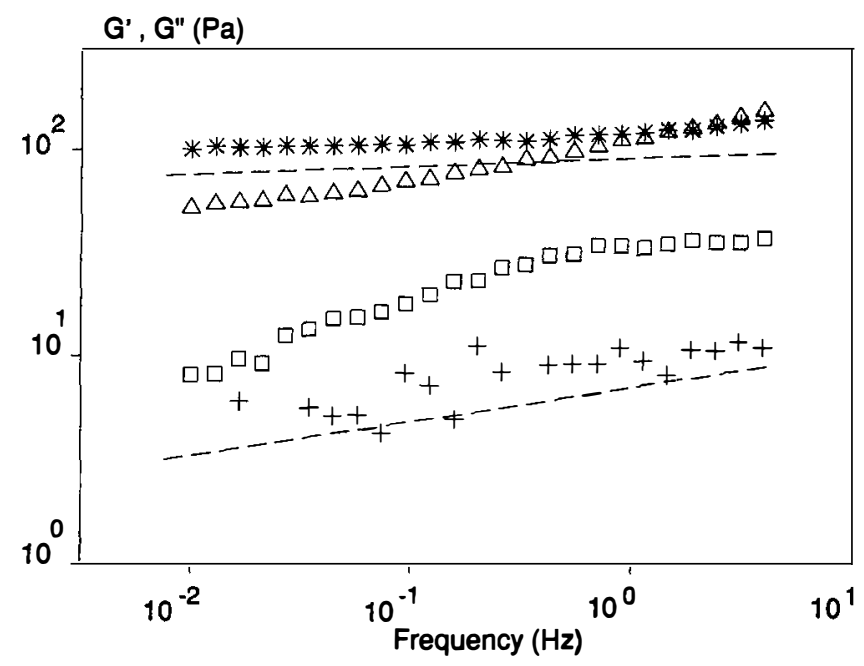

Fig. 8. Mechanical spectra of $15 \mathrm{~h}$ old starch/gum gels. Dashed lines, starch (concentration, $4 \%$ ). Starch $4 \%$ /guar gum $0.1 \%\left(*, G^{\prime} ;+, G^{\prime \prime}\right)$; starch $4 \%$ /guar gum $0.5 \%\left(\triangle, G^{\prime} ; \square, G^{\prime \prime}\right)$. Temperature, $25^{\circ} \mathrm{C}$.

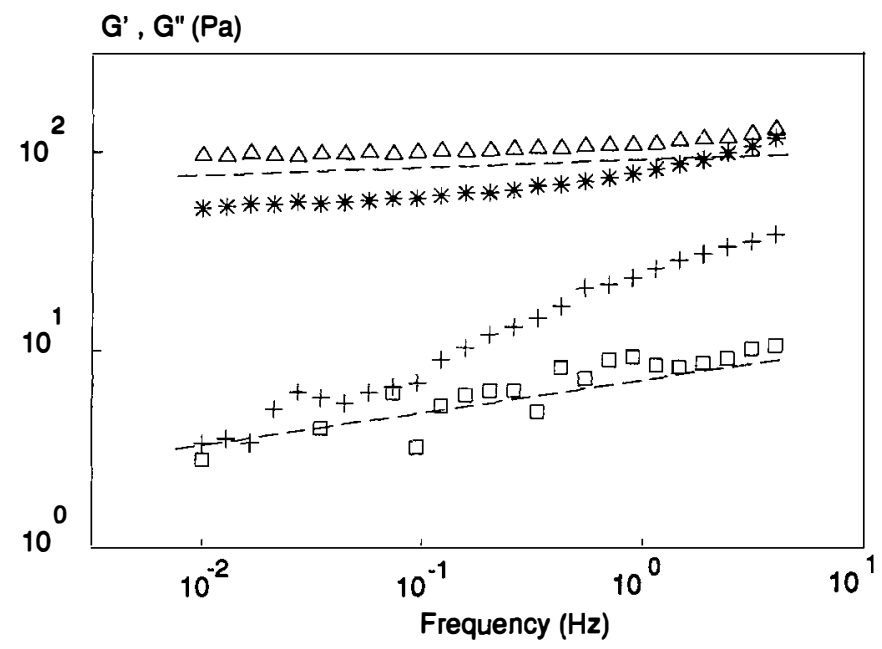

Fig. 9. Mechanical spectra of $15 \mathrm{~h}$ old starch/locust bean gum gels. Dashed lines, starch (concentration, $4 \%$ ). Starch $4 \% /$ locust bean gum $0.1 \%\left(\triangle, G^{\prime} ; \square, G^{\prime \prime}\right)$; starch $4 \% /$ locust bean gum $0.5 \%\left(*, G^{\prime} ;+, G^{\prime \prime}\right)$. Temperature, $25^{\circ} \mathrm{C}$.

range $(0.01-0.3 \mathrm{~Hz})$. However, the tangent of the loss angle $\delta\left(\operatorname{tg} \delta=G^{\prime \prime} / G^{\prime}\right)$ increased from 0.1 to $\sim 0.3$ when frequency ranged from 0.01 to $4 \mathrm{~Hz}$. This clearly means that the mixed system displayed a viscoelastic character which was more pronounced than in the gel with starch alone. It was also found that the $G^{\prime}$ versus frequency curve of the mixed system crossed that of starch alone illustrating the fact that a comparison of $G^{\prime}$ values at a given frequency (e.g. 


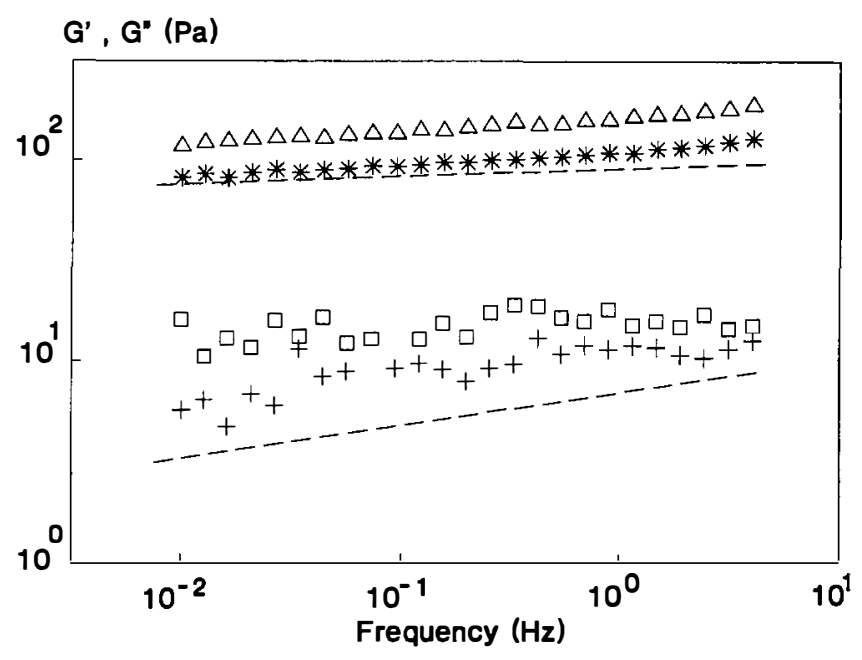

Fig. 10. Mechanical spectra of 15 h old starch/xanthan gum gels. Dashed lines, starch (concentration, $4 \%)$. Starch $4 \% / x a n t h a n$ gum $0.1 \%\left(*, G^{\prime} ;+, G^{\prime \prime}\right)$; starch $4 \% / x a n t h a n$ gum $0.5 \%\left(\triangle, G^{\prime} ; \square, G^{\prime \prime}\right)$. Temperature, $25^{\circ} \mathrm{C}$.

$1 \mathrm{~Hz}$ as in Figure 7) may yield erroneous interpretations since $G^{\prime}$ of the blend was lower than that of starch alone at $0.01 \mathrm{~Hz}$ and higher at $4 \mathrm{~Hz}$. For locust bean gum, similar observations as with guar could be made (Figure 9). The mixtures differed from starch alone by the $G^{\prime}$ versus frequency variations with slopes of 0.1 and 0.4 for $G^{\prime}$ and $G^{\prime \prime}$ respectively, and also by the higher $G^{\prime \prime} / G^{\prime}$ ratio. Similar conclusions relative to the viscoelastic character can thus be drawn for mixtures with guar and locust bean gum. The results with xanthan gum are shown in Figure 10. Surprisingly, the large differences which had been seen at $60^{\circ} \mathrm{C}$ (see Figure 3 ) were not confirmed. The $G^{\prime}$ value for $0.1 \%$ xanthan did not differ very much from that of starch alone, namely 110 versus $90 \mathrm{~Pa}$ at $1 \mathrm{~Hz}$, while it was slightly higher $(165 \mathrm{~Pa})$ at $0.5 \%$ xanthan. Here also, we found an increase of the slope of the $G^{\prime}$ versus frequency trace, namely 0.07 for the mixed system compared with 0.03 for starch alone.

\section{Discussion}

The results obtained for starch alone show that the pastes at $60^{\circ} \mathrm{C}$ are markedly elastic, the storage modulus considerably exceeding the loss modulus while $G^{\prime}$ exhibits a low frequency-dependence. These results are consistent with previous data on maize starch pastes $(10-12)$. The $G^{\prime \prime} / G^{\prime}$ ratio had been reported in the range $0.05-0.13$; in the present work, this ratio was $\sim 0.15$ at $1 \mathrm{~Hz}$, but the concentration we investigated was low $(4 \%)$ relative to those in the abovementioned references $(6-10 \%)$. Moreover, the preparation procedures were not similar and the frequency measurements were performed in different conditions. In any case, it is clear that maize starch pastes exhibit the viscoelastic behaviour typical of a 'weak' gel (9). 
The mechanical spectrum of the maize starch gel at $25^{\circ} \mathrm{C}$ is also consistent with previously reported data $(12-14)$. The $G^{\prime \prime} / G^{\prime}$ ratio was $\sim 0.05$ and the storage modulus was almost independent of frequency. These two features reflect the properties of a 'true' gel (9). It is noteworthy that the mechanical spectrum of the gel aged for $15 \mathrm{~h}$ at $25^{\circ} \mathrm{C}$ does not differ very much from the spectrum displayed by the hot paste at $60^{\circ} \mathrm{C}$ except that the absolute values of the moduli are higher. This reflects a similarity in the structure of the two systems. A maize starch paste can be described as a suspension of swollen gelatinized granules dispersed in a macromolecular medium. The viscoelastic properties of such a system are known to be governed by the characteristics of the dispersed phase as well as the continuous phase. The relative importance of both phases depends mainly upon the starch concentration $(5,6,15-17)$. From swelling-solubility experiments in HR-LS conditions which are the same as in the present work, we estimated (4) the volume fraction of the dispersed phase to be 0.68 and the starch concentration in the continuous phase to be $2.7 \%$, amylose being the major component $(\sim 2.2 \%)$. The rheology of suspensions with such a high volume fraction of swollen particles is known to be strongly influenced by the dispersed phase. The continuou's phase makes an additional contribution the importance of which depends upon its own viscoelastic properties. In the present case the low starch concentration of the continuous phase makes its rheology negligible and that of the paste at $60^{\circ} \mathrm{C}$ primarily governed by the dispersed phase.

Similarly, the starch gel which is formed upon cooling to $25^{\circ} \mathrm{C}$ for $15 \mathrm{~h}$ can be regarded as a composite $(15,16,18)$. The swollen granules are embedded within a gel matrix which results from amylose gelation. The gelation process is quite slow as could be observed from the kinetics experiments. This can be due to the relatively low amylose content $(2.2 \%)$ of the continuous phase. The similarity between the mechanical spectra at $60^{\circ} \mathrm{C}$ on one side, and the ones obtained after ageing for $15 \mathrm{~h}$ at $25^{\circ} \mathrm{C}$ on the other, indicates that swollen particles make a major contribution to the viscoelastic behaviour of starch systems in the hot paste and in the gel state. This does not mean, however, that the influence of amylose in the gel properties is negligible. Rather, this component is responsible for the reinforcement of the elastic character of the system which is expressed in the higher $G^{\prime}$ and $G^{\prime \prime}$ moduli, the lower $G^{\prime \prime} / G^{\prime}$ ratio and the slight frequency dependence of $G^{\prime}$.

Incorporation of an hydrocolloid in such maize starch systems yields unexpected results in the hot paste as well as in the gelled state. We demonstrated (4) that the added polysaccharide is located and concentrated within the continuous phase but does not influence the swelling process. For a total hydrocolloid content ranging from 0.1 to $0.5 \%$ the actual hydrocolloid concentration in the continuous phase is expected to vary from 0.31 to $1.53 \%$, the volume fraction available to the soluble macromolecules being 0.32 . Addition of xanthan gum resulted, for the hot paste, in a simple shift of the $G^{\prime}$, $G^{\prime \prime}$ versus frequency curves; moreover, the $G^{\prime}$ variation with xanthan concentration was approximately linear. This effect can simply be ascribed to a modification of the continuous phase when the viscoelastic properties are 
dramatically changed by the presence of xanthan gum known to exhibit 'weak' gel properties $(19,20)$. This leads to a reinforcement of the overall properties of the suspension. More subtle changes were observed in the hot pastes with guar gum and locust bean gum. A shift of $G^{\prime \prime}$ to higher values was noticed while $G^{\prime}$ did not vary very much. Moreover, the storage modulus had become very slightly, but significantly, frequency-dependent. These two features indicate that the elastic character of the system was attenuated. The elastic character of galactomannan solutions is far less marked than that of xanthan gum. This may provide a straightforward explanation for the limited effect of the galactomannan incorporation on the storage modulus. However, the fact that a maximum in $G^{\prime}$ is seen as the galactomannan content increases (Figure 4) also suggests that other phenomena are involved in the process. One assumption would be that this effect is the result of incompatibility phenomena between the amylose component and the galactomannan. Incompatibility between chemically dissimilar polymers is known to be a general rule for thermodynamic reasons. Although there is scant literature on the phase behaviour of biopolymer mixtures, immiscibility is expected to take place in many cases, giving rise to two-phase systems (21). This has been described for amylose/dextran and amylose/amylopectin at $70^{\circ} \mathrm{C}$, resulting in a macroscopic phase separation $(7,8)$. A similar process has been observed for amylose/guar mixtures at $70^{\circ} \mathrm{C} \mathrm{(22).} \mathrm{In}$ that case, however, the macroscopic phase separation could not be completed due to the high viscosity of the gum which hindered total separation of the phases. The consequences of such a phase separation in such an intricate system as a starch/hydrocolloid mixture are difficult to predict. However, this interpretation provides an attractive basis for explaining the viscoelastic properties of starch/hydrocolloid hot pastes. As in pastes with starch alone the viscoelastic properties of the starch/hydrocolloid pastes are primarily governed by the dispersed phase of the suspension. The added hydrocolloid plays an additional role which is either pronounced as with xanthan gum (due to the gelling ability of this polysaccharide) or more subtle as with the galactomannans owing to their poor gelling ability. The main effect in that case is an increase in the viscosity of the continuous medium due to the thickening properties of these polysaccharides.

The results from gel-cure experiments and the mechanical spectra of $15 \mathrm{~h}$-old gels can be interpreted on a similar basis. As with starch alone the properties are primarily governed by the dispersed phase, but an additional role is played by amylose, the gelation of which is effected by the added hydrocolloid. Although the process is accelerated in a first step, as estimated from the initial $G^{\prime}$ increase, the final firmness of the mixed gels does not appear to differ very much from that of gels of starch alone. Furthermore, the mechanical spectra of the starch/ hydrocolloid gels show that the elastic character is attenuated as compared with starch. These effects are consistent with results of preliminary experiments on amylose/hydrocolloid mixtures (22). Amylose gelation was accelerated by the incorporation of another polysaccharide but the final gel properties were dramatically depressed. Here also, these effects were interpreted on the basis of incompatibility phenomena. The more rapid initial increase of gel firmness was 
induced by the presence of the added polysaccharide and was related to a phase separation, as discussed above, resulting from incompatibility between unlike polymers, i.e. the amylose component and the hydrocolloid. This process did not give rise, however, to a macroscopic phase separation due to the high viscosity of the medium accentuated by the gelation of amylose. Since the overall separation process could not be completed, the amount of amylose involved in the gel network was lower than in gels of amylose alone, resulting in a lower rigidity than expected. We suggest that similar phenomena take place in starch/hydrocolloid systems. However, in that case, the dispersed phase makes the essential contribution to the overall rheology of the system, amylose gelation only playing an additional role. It is thus clear that although less dramatically modified than expected from viscometric measurements (5), the viscoelastic properties of the starch/hydrocolloid systems as investigated in the present work are markedly different from the properties of gels simply obtained with starch alone.

\section{Conclusions}

Starch concentration was chosen at this quite low value (4\%) in order to describe the starch dispersion on a simple basis. Furthermore, only one preparation procedure was investigated. This means that the present results cannot be extended to other experimental conditions without additional experiments. It would be particularly helpful to investigate higher concentrations and other types of starches. The overall phenomena, however, should be the same. One of the most important results of the present study is that the incorporation of another polysaccharide to a starch system does not necessarily result in a dramatic rise of the elastic modulus as would be expected by assuming that the properties were additive. Despite the viscosity of the pastes from a blend of a starch and an hydrocolloid may be markedly increased, the viscoelastic properties are less dramatically affected; the changes are more subtle. The modifications which result from the addition of hydrocolloids to a starch system are thus far more complex than expected and they must be considered in the light of the properties of blended immiscible synthetic polymers, particularly through thermodynamic approaches.

\section{Acknowledgements}

$\mathrm{MC}$ is indebted to Sanofi Bio Industrie for financial support and the authors wish to thank Mrs G.Llamas for her technical assistance.

\section{References}

1. Sandstedt,R.M. and Abbott,R.C. (1964) Cereal Sci. Today, 9, 13-26.

2. Christiansson,D.D., Hodge,J.E., Osborne,D. and Detroy,R.W. (1981) Cereal Chem., 58, 513517.

3. Sajjan,S.U. and Rao,M.R.R. (1987) Carbohydr. Polym., 7, 395-402.

4. Alloncle,M., Lefebvre,J., Llamas,G. and Doublier,J.L. (1989) Cereal Chem., 64, 21-26.

5. Doublier,J.L., Llamas,G. and Le Meur,M. (1987) Carbohydr. Polym., 7, 251-275.

6. Ellis,H.S., Ring,S.G. and Whittam,M.A. (1989) J. Cereal Sci., 10, 33-44. 
7. Kalichesvky,M.T., Orford,P.D. and Ring,S.G. (1986) Carbohydr. Polym., 6, 145-154.

8. Kalichesvky,M.T. and Ring,S.G. (1987) Carbohydr. Res., 162, 323-328.

9. Clark,A.H. and Ross-Murphy,S.B. (1987) Adv. Polym. Sci., 83, 57-192.

10. Evans,I.D. and Haisman,D.R. (1979) J. Text. Stud., 17, 253-265.

11. Wong,R.B.K. and Lelievre,J. (1981) Rheol. Acta, 20, 299-307.

12. Eliasson,A.C. (1986) J. Text. Stud., 17, 253-265.

13. Lindahl,L. and Eliasson,A.C. (1986) J. Sci. Food Agric., 37, 1125-1132.

14. Eliasson,A.C., Finstad,H. and Ljunger,G. (1988) Die Stärke, 37, 95-100.

15. Ring,S.G. (1985) Die Stärke, 37, 80-83.

16. Miles,M.J., Morris,V.J., Orford,P.D. and Ring,S.G. (1985) Carbohydr. Res., 135, 271-281.

17. Doublier,J.L. (1987) J. Cereal Sci., 5, 247-262.

18. Ring,S.G. and Stainsby,G. (1982) Progr. Food Nutr. Sci., 6, 323-329.

19. Frangou,S.A., Morris,E.R., Rees,D.A., Richardson,R.K. and Ross-Murphy,S.B. (1983) J. Polym. Sci., Polym. Letters Eds, 20, 531-538.

20. Ross-Murphy,S.B., Morris,V.J. and Morris,E.R. (1983) Faraday Symp. Chem. Soc., 18, 115129.

21. Albertsson,P.A. (1962) Methods Biochem. Anal., 10, 229-262.

22. Alloncle,M. (1990) PhD Thesis, Université Paris-Sud. 